DiRECTEUR DE LA PUBLICATION / PUBLICATION DIRECTOR: Bruno David,

Président du Muséum national d'Histoire naturelle

RÉDACTEUR EN CHEF / EDITOR-IN-CHIEF : Jean-Philippe Siblet

ASSISTANTE DE RÉDACTION / ASSISTANT EDITOR: Sarah Figuet (naturae@mnhn.fr)

Mise EN PAGE / PAGE LAYOUT: Sarah Figuet

COMITÉ SCIENTIFIQUE / SCIENTIFIC BOARD:

Luc Abbadie (UPMC, Paris)

Luc Barbier (Parc naturel régional des caps et marais d'Opale, Colembert)

Aurélien Besnard (CEFE, Montpellier)

Vincent Boullet (Expert indépendant flore/végétation, Frugières-le-Pin)

Hervé Brustel (École d'ingénieurs de Purpan, Toulouse)

Patrick De Wever (MNHN, Paris)

Thierry Dutoit (UMR CNRS IMBE, Avignon)

Éric Feunteun (MNHN, Dinard)

Romain Garrouste (MNHN, Paris)

Grégoire Gautier (DRAAF Occitanie, Toulouse)

Olivier Gilg (Réserves naturelles de France, Dijon)

Frédéric Gosselin (Irstea, Nogent-sur-Vernisson)

Patrick Haffner (UMS PatriNat, Paris)

Frédéric Hendoux (MNHN, Paris)

Xavier Houard (OPIE, Guyancourt)

Isabelle Leviol (MNHN, Concarneau)

Francis Meunier (Conservatoire d'espaces naturels - Hauts-de-France, Amiens)

Serge Muller (MNHN, Paris)

Francis Olivereau (DREAL Centre, Orléans)

Laurent Poncet (UMS PatriNat, Paris)

Nicolas Poulet (OFB, Vincennes)

Jean-Philippe Siblet (UMS PatriNat, Paris)

Laurent Tillon (ONF, Paris)

Julien Touroult (UMS PatriNat, Paris)

COUVERTURE / COVER:

Pavage de Corbicules. Crédit photo: C. Lemarchand.

\section{MUSÉuM * Nantes

Les Publications scientifiques du Muséum publient aussi / The Museum Science Press also publish:

Adansonia, Zoosystema, Anthropozoologica, European Journal of Taxonomy, Geodiversitas, Cryptogamie sous-sections Algologie, Bryologie, Mycologie, Comptes Rendus Palevol.

Diffusion - Publications scientifiques Muséum national d'Histoire naturelle

CP $41-57$ rue Cuvier F-75231 Paris cedex 05 (France)

Tél.: 33 (0)1 40794805 / Fax: 33 (0)1 40793840

diff.pub@mnhn.fr / http://sciencepress.mnhn.fr

(C) Publications scientifiques du Muséum national d'Histoire naturelle, Paris, 2020

ISSN (électronique / electronic): 1638-9387 


\title{
Utilisation des Corbicules (Corbicula sp. Müller, 1774) comme biomarqueurs d'éléments toxiques dans les réseaux trophiques aquatiques en France
}

\author{
Charles LEMARCHAND \\ Muséum Henri-Lecoq, 15 rue Bardoux, F-63000 Clermont-Ferrand (France) \\ clemarchand@clermontmetropole.eu \\ René ROSOUX \\ Conservatoire d'Espaces naturels Centre-Val de Loire, \\ 3 rue de la Lionne, F-45000 Orléans (France) \\ siege.orleans@cen-centrevaldeloire.org
}

Philippe BERNY

VetAgro Sup, universités Clermont Auvergne et Lyon, Laboratoire de Toxicologie vétérinaire, 1 avenue Bourgelat, F-69280 Marcy l'Etoile (France)

philippe.berny@vetagro-sup.fr

Soumis le 10 juillet 2019 | Accepté le 8 novembre 2019 | Publié le 27 novembre 2020

MOTS CLÉS

Corbicula fluminea Corbicula fluminalis toxicologie.

\begin{abstract}
Lemarchand C., Rosoux R. \& Berny P. 2020. - Utilisation des Corbicules (Corbicula sp. Müller, 1774) comme biomarqueurs d'éléments toxiques dans les réseaux trophiques aquatiques en France, in Léonard L. (éd.), Colloque national de malacologie continentale, Nantes, 6 et 7 décembre 2018. Naturae 2020 (14): 213-218. https://doi.org/10.5852/naturae2020a14

\section{RÉSUMÉ}

Les Corbicules, et notamment la Corbicule asiatique (Corbicula fluminea (O. F. Müller, 1774)) ont, ces dernières années, très rapidement colonisé plusieurs grands bassins hydrographiques en France (Loire, Garonne, Rhône) et en Europe, comme dans d'autres foyers d'introduction. Leur développement exponentiel et leurs capacités de filtration ne sont pas sans conséquence sur la charge particulaire de ces milieux, et peuvent au moins localement entraîner des phénomènes de concurrence ou de compétition vis-à-vis des autres Mollusques filtreurs autochtones. Au-delà de leur effet d'organismes filtreurs, ces espèces ont par ailleurs été intégrées aux réseaux trophiques, devenant la proie d'espèces aussi diverses que certains poissons (Silure glane Silurus glanis (Linnaeus, 1758)), Oiseaux (Limicoles notamment), Mammiferes (Rat musqué Ondatra zibethicus (Linnaeus, 1766), Raton laveur Procyon lotor (Linnaeus, 1758)) ou encore Crustacés (écrevisses). Elles sont consommées vivantes ou exploitées à l'état de cadavres lors d'épisodes de mortalité massive. Ces dernières années, les Corbicules ont été intégrées à plusieurs travaux de recherche et de terrain. Une partie d'entre eux, financés par le volet Recherche/Données/Informations du Plan Loire Grandeur Nature et coordonnés par le Muséum d'Orléans, ont valorisé les Corbicules en tant que biomarqueurs de la contamination de la faune aquatique par des composés toxiques (pesticides, métaux, anticoagulants, radioéléments) captés par filtration et transmis, par les prédations successives, à l'ensemble des espèces présentes.
\end{abstract}

\section{ABSTRACT}

Use of Asian clams (Corbicula sp. Müller, 1774) as biomarkers of toxic compounds in aquatic food webs in France.

In recent years, Asian clams and especially Corbicula species (C. Aluminea (O. F. Müller, 1774) have widely and rapidly colonized several large river basins in France (Loire, Garonne, Rhône) and in Europe, as in other loci of introduction. Their exponential development and their filtration capacities have impact on environments, and can at least locally cause competition between na- 
KEY WORDS

Corbicula fluminea,

Corbicula fluminalis, Toxicology. tive and introduced species. Beyond their effect of filtering organisms, Asian clams have also been integrated into aquatic food webs, becoming the prey of species as diverse as fishes (Catfish Silurus glanis (Linnaeus, 1758)), birds (wader species), mammals (Muskrat Ondatra zibethicus (Linnaeus, 1766), Raccoon Procyon lotor (Linnaeus, 1758)) or crustaceans (crayfish). They are consumed alive or exploited as corpses during episodes of mass mortality. In recent years, Asian clams were used by research and field work. Some of them, funded by the Research / Data / Information component of the Loire Grandeur Nature Plan and coordinated by the Orléans Museum, have promoted Asian clams as biomarkers of aquatic fauna contamination by toxic compounds (pesticides, metals, anticoagulants, radio elements) collected by filtration and transmitted by successive predations to the rest of the aquatic food web.

\section{INTRODUCTION}

L'introduction d'espèces allochtones constitue désormais la deuxième principale menace pesant sur la biodiversité, après la destruction des habitats. La quasi-totalité des systèmes naturels de la biosphère est concernée par ce phénomène (Schmitz \& Simberloff 1997; Genovesi \& Shine 2004). Les ydrosystèmes français ne sont évidemment pas épargnés et, parmi les espèces animales rencontrées, les Mollusques envahissants occupent une place importante, puisqu' ils peuvent constituer près de la moitié de l'ensemble des espèces non indigènes recensées (Devin et al. 2005; Pascal et al. 2006).

Les Corbicules (Cyrenidae Gray, 1840) originaires d'Asie du Sud-Est, ont été introduites en Europe par les activités humaines, notamment par le transport maritime. Parmi les espèces recensées sur le continent européen (Corbicule striolée Corbicula fluminalis (O. F. Müller, 1774), Corbicule asiatique Corbicula fluminea (O. F. Müller, 1774) et Corbicule japonaise Corbicula leana (Prime, 1867)), l'espèce la plus communément citée est la Corbicule asiatique $C$. $f u-$ minea (Prié 2017).

Les Corbicules disposent d'une grande capacité de reproduction et de dispersion. Ces espèces envahissantes à stratégie de type $\mathrm{R}$ présentent une croissance rapide, une maturité sexuelle précoce, une courte durée de vie et une fécondité élevée, pouvant se reproduire par androgenèse. Elles semblent cependant parfois moins résistantes aux pollutions que certains Mollusques autochtones (Faria et al. 2010).

En dépit des impacts négatifs que ces espèces peuvent causer de par leur caractère fortement invasif, les Corbicules constituent des biomasses localement importantes et ainsi représentent une ressource alimentaire conséquente pour bon nombre de prédateurs (Simon \& Garnier-Laplace 2005; Tablado et al. 2010). De plus, en raison de leur capacité à la bioaccumulation des polluants, les Corbicules constituent de très bons indicateurs biologiques et sont régulièrement utilisées dans la biosurveillance des milieux aquatiques (Sousa et al. 2008; Jou et al. 2009). En effet, les Corbicules filtrent de grandes quantités d'eau (10 l/individu/jour) (Simon et al. 2000) et peuvent accumuler les polluants dans leurs tissus à une concentration 103 à 104 fois plus importante que celle contenue dans l'eau ambiante (Jou et al. 2009).

\section{MATÉRIELS ET MÉTHODES}

Porté par VetAgro Sup Campus Vétérinaire de Lyon et coordonné entre 2009 et 2015 par C. Lemarchand, R. Rosoux et P. Berny, un programme concerté de recherches sur le bassin de la Loire a été mené. Il a consisté en l'étude écotoxicologique d'espèces indicatrices, afin de mettre en évidence la bioaccumulation et la bioamplification des polluants dans l'ensemble du réseau trophique du fleuve (Lemarchand et al. 2013a, 2015). Ainsi, trois espèces super prédatrices (Loutre d'Europe Lutra lutra Linnaeus, 1758; Balbuzard pêcheur Pandion haliaetus Linnaeus, 1758; Grand cormoran Phalacrocorax carbo Linnaeus, 1758), trois Poissons polyphages (Poisson chat Ameiurus melas Rafinesque, 1820; Chevaine Squalius cephalus Linnaeus, 1758; Carassin Carassius carassius Linnaeus, 1758), trois Crustacés (Écrevisses de Louisiane Procambarus clarkii Girard, 1852; de Californie Pacifastacus leniusculus Dana, 1852; Américaine Orconectes limosus Rafinesque, 1817) ainsi que des Bivalves (dont les Corbicules Corbicula sp.) ont été étudiés.

Pour réaliser cette étude, neuf sites de prélèvements, représentatifs du fonctionnement du bassin ligérien et permettant l'échantillonnage d'un maximum d'individus des espèces retenues, ont été déterminés: des têtes de bassins à l'estuaire, en passant par les plaines alluviales (Fig. 1). Ainsi nous avons sélectionné un site dans chacun des secteurs des linéaires de la Loire et de son principal affluent, l'Allier, c'est-à-dire au niveau de l'estuaire (9), en Loire aval (amont de Nantes, 8), en Loire moyenne (Val d'Orléans, 6), au Bec d'Allier (confluence entre la Loire et l'Allier, 5), en Loire amont (aval du barrage de Grangent, 4), Loire supérieure (amont du Puy-en-Velay, 1), Allier des plaines (près de Vichy, 3) et Allier amont (agglomération clermontoise, 2). Il nous a également paru pertinent de rajouter un point de prélèvement sur la Vienne afin de contribuer aux études locales après l'effacement du barrage de Maisons Rouges en 1998.

Pour chaque site pour lequel le prélèvement s'est avéré possible, environ $200 \mathrm{~g}$ de tissus de Corbicules ont été récoltés: à partir d'un prélèvement de moules entières effectué sur le terrain, la partie charnue seule en a été rapidement extraite et a constitué l'échantillon analysé, l'objectif de l'étude étant d'évaluer le pool de contaminants transmis aux prédateurs de ces espèces, dont bien peu consomment les coquilles (à l'excep- 
tion de certains poissons comme le Silure glane, Silurus glanis (Linnaeus, 1758) (Corvi et al. 2005). Les tissus extraits ont été conservés au congélateur.

Plus d'une cinquantaine (54) de composés toxiques différents ont été recherchés par chromatographie gazeuse, liquide ou par spectrométrie de masse, parmi lesquels des pesticides (organochlorés, organophosphorés, herbicides, pyréthrines, carbamates et anticoagulants), des métalloïodes (plomb, mercure, arsenic, cuivre, cadmium) et 16 congénères de polychlorobiphényles (PCBs). Le protocole de suivi, servant de standard aux analyses toxicologiques de la faune sauvage (réseau SAGIR de l'ONCFS) a été largement étudié et éprouvé au sein de VetAgro Sup ces dernières années (Mazet et al. 2005; Lemarchand 2007; Lemarchand et al. 2010, 2011, 2012). Les échantillonnages de terrain ont pu permettre de constituer des lots d'analyses complets et exploitables pour les sites situés sur l'Allier (site de l'amont, $\mathrm{n}^{\circ} 2$ sur la Figure 1, et de Vichy, site $\mathrm{n}^{\circ} 3$ ), sur la Loire (site de l'amont, $\mathrm{n}^{\circ} 4$ et du Val d'Orléans, $n^{\circ} 6$ ) ainsi que sur la Vienne (site $n^{\circ} 7$ ).

\section{RÉSULTATS ET DISCUSSION}

Sur les huit pesticides organochlorés analysés, seuls le lindane, l'endosulfan et le DDE ont été détectés systématiquement dans les tissus des Corbicules, les deux premiers constituant les deux composés les plus abondants (Fig. 2). Le DDT a également été retrouvé dans le bassin ligérien, mais de façon plus épisodique dans les Corbicules issues du secteur de Vichy et de la Vienne. Cette mention peut suggérer une utilisation récente de ce composé (interdit en France en 1973, c'est-à-dire avant les premières mentions de présence des Corbibules), mais aussi sa forte rémanence dans les sédiments des grands cours d'eau ou des barrages, d'où il peut être remis en suspension lors de grandes crues ou de vidanges (Díaz-Barriga et al. 2003). Les concentrations en pesticides organochlorés sont assez similaires sur l'ensemble des sites échantillonnés (pvalue $=0,0822$ ) et correspondent à des concentrations que l'on retrouve dans d'autres cours d'eau européens (Chevreuil et al. 1996 ; Berny et al. 2003). En ce qui concerne les PCBs analysés, exprimés ici sous forme du total des concentrations des 16 congénères recherchés dans cette étude, des teneurs assez comparables ont été relevées sur les divers secteurs du bassin hydrographique $(\mathrm{p}$-value $=0,06269)$. La moyenne des concentrations en PCBs sur l'ensemble du bassin avoisine les $2,45 \mathrm{mg} / \mathrm{kg}$ de lipides, ce qui en fait donc le composé organochloré le plus majoritairement rencontré sur la Loire et ses affluents. Cette contamination se situe dans la même gamme que celle de la Seine $(0,635$ à $3,9 \mathrm{mg} / \mathrm{kg}$ de poids sec; Chevreuil et al. 1996) mais est nettement inférieure à celle établie sur le Pô en Italie, où les concentrations ont atteint 17,230 mg/kg de lipides (Binelli et al. 2001).

Aucun autre pesticide organochloré, ni aucun pesticide organophosphoré, carbamate, herbicide, pyréthrine ou anticoagulant n'a été détecté dans les tissus des Corbicules au cours de cette étude. Certains d'entre eux ont cependant

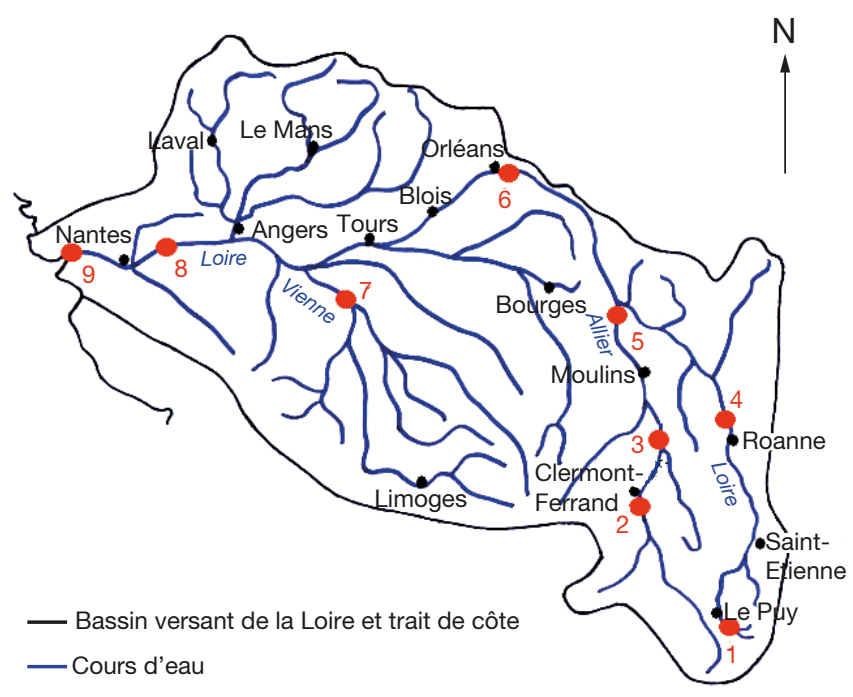

FIG. 1. - Localisation des neuf sites de prélèvements sur le bassin versant de la Loire et de ses affluents.

pu être mesurés, mais de façon anecdotique, dans les tissus de cormorans et de loutres au cours de cette même étude (Lemarchand et al. 2014; Alomar et al. 2016).

Pour certains d'entre eux (pesticides organophosphorés, pyréthrines), cela peut être dû à leur courte demi-vie, à leurs caractères faiblement persistants ou peu accumulatifs dans les environnements et les réseaux trophiques (Gitahi et al. 2002), ou encore à des propriétés physico-chimiques particulières et une faible interaction avec l'activité de filtration de Mollusques comme les Corbicules. En tout état de cause, davantage d'investigations utilisant des Mollusques aquatiques sont requises afin de mieux cerner les modalités de l'accumulation de xénobiotiques comme les pesticides ou les PCBs.

À la différence des pesticides organochlorés, tous les métalloïdes qui ont été recherchés dans cette étude ont été retrouvés dans les tissus des Corbicules (Fig. 3). La contamination est quasi systématique dans tous les échantillons sur la totalité des secteurs, à l'exception de l'arsenic qui n'a pas été détecté dans les Corbicules de l'Allier amont. Le cuivre est, de loin, l'élément que l'on retrouve en plus grande quantité dans les tissus des Corbicules avec en moyenne $41,277 \mathrm{mg} / \mathrm{kg}$ de lipides, suivi par le cadmium $(2,165)$, le plomb $(1,412)$, l'arsenic $(0,846)$ et enfin le mercure $(0,317)$.

L'arsenic n'a pas été retrouvé sur le site de l'Allier amont, mais sur les autres secteurs, la contamination par ce composé est systématique quel que soit l'homogénat analysé et nettement plus élevée pour les individus collectés sur le site de la Vienne, les autres sites affichant des teneurs plus comparables (Fig. 3). Ces concentrations varient de 0,5 à $1,9 \mathrm{mg} / \mathrm{kg}$ de lipides et sont inférieures à celles rencontrées dans les Moules zébrées du Saint-Laurent au Canada (2,49-8,23 mg/kg de poids $\mathrm{sec})$, une des rares références concernant l'arsenic dans des Mollusques aquatiques en milieu naturel (Kwan et al. 2003).

La contamination au cadmium est globalement comparable pour l'ensemble de sites du bassin, tous les homogénats analysés étant systématiquement contaminés (Fig. 3). 


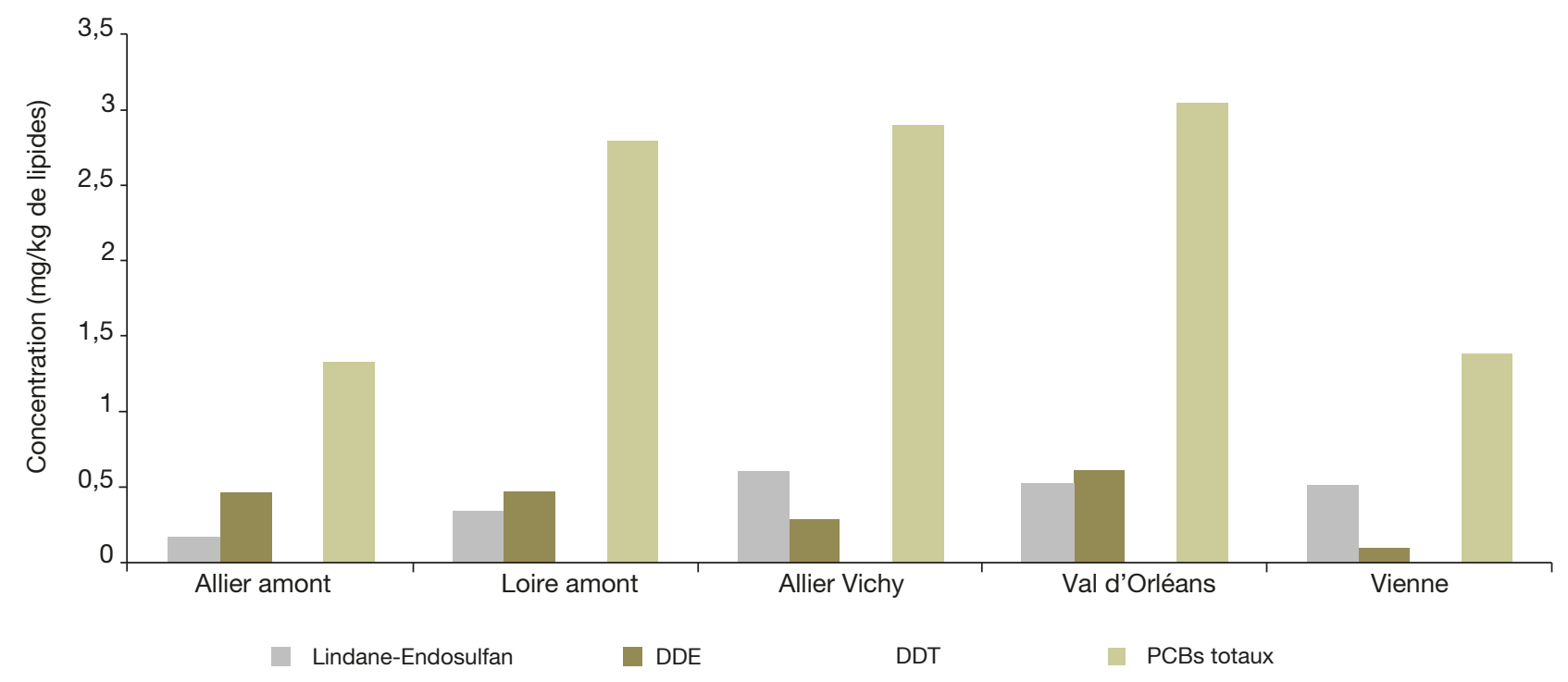

FIG. 2. - Distribution des composés organochlorés (pesticides organochlorés et PCBs) dans les tissus des Corbicules du bassin de la Loire. Abréviations: DDE, Dichlorodiphényldichloréthylène; DDT, Dichlorodiphényltrichloroéthylène; PCBs, Polychlorobiphényles totaux.

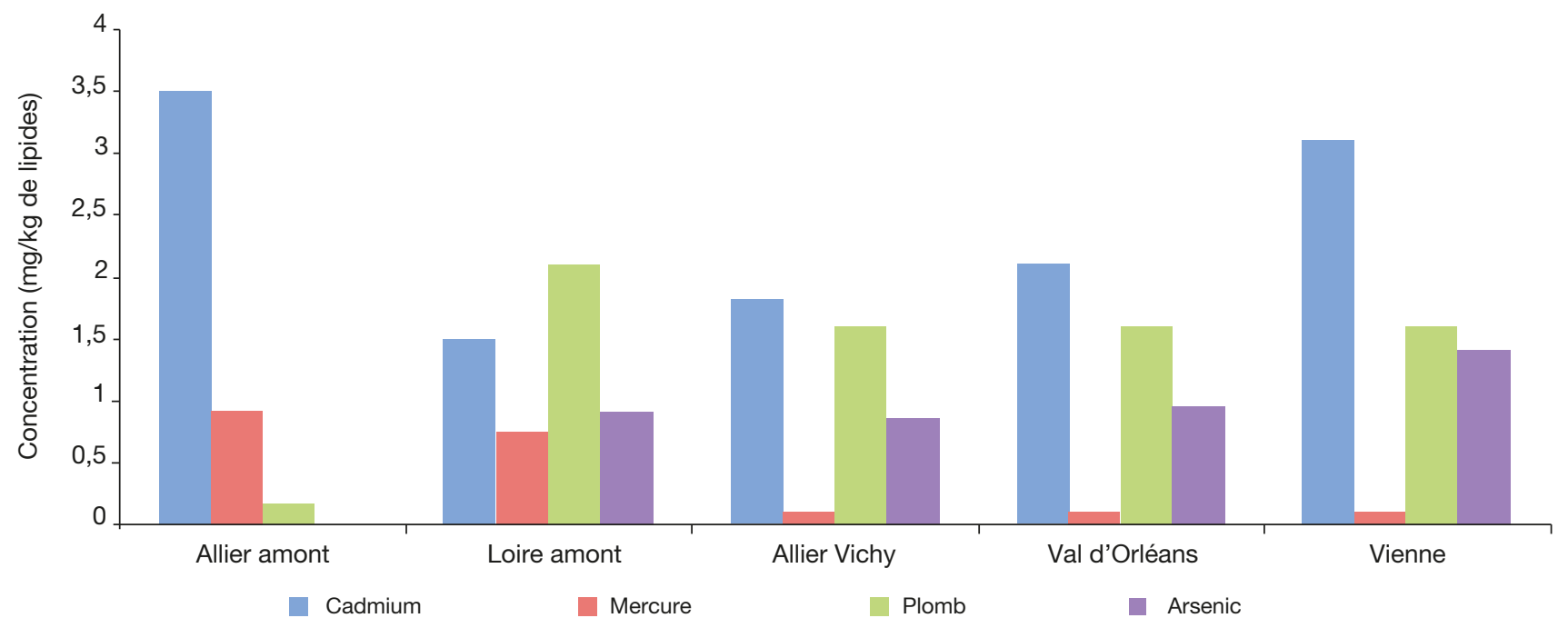

FIG. 3. - Distribution des métalloïdes dans les tissus des Corbicules du bassin de la Loire.

Ces valeurs sont en adéquation avec celles rencontrées dans la littérature. Ainsi sur le Riou Mort, petit affluent du Lot (France), les concentrations de ce composé dans les Corbicules sont de $4 \mathrm{mg} / \mathrm{kg}$ de poids frais (Marie et al. 2006). Sur le lac Léman (France) elles varient de 1,2 à 4,1 mg/kg de poids sec (Corvi et al. 2005), sur le Saint-Laurent (Canada) de 1,8 à $7,43 \mathrm{mg} / \mathrm{kg}$ de poids sec (Kwan et al. 2003) et sur le lac Majeur (Italie) la concentration est de 3,44 mg/kg de poids sec dans les Moules zébrées (Camusso et al. 2001).

Les concentrations en plomb relevées dans les Corbicules sont significativement inférieures en Allier amont en comparaison des quatre autres sites qui eux, montrent des valeurs relativement similaires (Fig. 3). Elles s'échelonnent entre 0 et $2,9 \mathrm{mg} / \mathrm{kg}$ de lipides et sont comparables à d'autres sites français ou canadiens où les Moules zébrées ont servi de bio-indicateurs
(Berny et al. 2003; Kwan et al. 2003; Corvi et al. 2005). Sur la Seine, ces valeurs sont plus élevées et peuvent aller jusqu'à $25 \mathrm{mg} / \mathrm{kg}$ de poids sec (Chevreuil et al. 1996).

Si la contamination en arsenic est absente du site Allier amont et que celles du cuivre et du plomb y sont nettement inférieures aux autres sites, il en est tout autrement pour la contamination par le mercure (Fig. 3). Celle-ci est plus élevée sur l'Allier amont que sur les quatre autres secteurs du bassin. Les concentrations sont également nettement supérieures sur le site de la Loire amont que sur le site de Vichy, du Val d'Orléans ou de la Vienne, qui montrent des contaminations analogues (de 0,06 à $1,1 \mathrm{mg} / \mathrm{kg}$ de lipides). Ces valeurs sont inférieures à celles rencontrées en Espagne $(1,8-3 \mathrm{mg} / \mathrm{kg}$ de poids sec dans les Corbicules et 2,2 à 5,7 dans les Unionidae; Faria et al. 2010), ou dans les Moules zébrées du 
Canada (0,103-2,2 mg/kg de poids sec; Kwan et al. 2003). Ces valeurs élevées de concentrations en mercure dans les tissus ont été également observées chez les loutres du même secteur (Lemarchand et al. 2010), confirmant une accumulation locale et une contamination relativement importante des réseaux trophiques. La plupart des espèces animales étudiées se trouvant toutefois dans une dynamique de population stable ou en expansion (Lemarchand et al. 2013a, b; Prié 2017; Rosoux \& Lemarchand 2019), l'impact de la contamination sur la conservation des espèces semble toutefois relativement limité, au moins à court terme.

\section{CONCLUSION}

La contamination des Corbicules du bassin de la Loire par les composés organochlorés ou les métalloïdes est donc fréquente à quasi-systématique, des variations locales étant par ailleurs mises en évidence. En revanche, certains autres composés toxiques, comme certains pesticides, ne sont pas identifiés dans les tissus des Corbicules, à la différence des mesures effectuées au sein des tissus d'autres espèces animales sur ce même bassin. Il apparaît donc, dans certaines limites analytiques et écologiques, que les Corbicules peuvent constituer un bioindicateur, renseignant sur un degré de contamination des habitats naturels. Dans la mesure où elles constituent un matériel biologique représentant une biomasse importante et facilement accessible, l'intérêt de leur utilisation s'en voit renforcé. D'autres études sont cependant nécessaires afin, d'une part, d'évaluer la diversité totale des contaminants que les Corbicules permettraient de révéler et d'autre part, d'estimer le transfert de ces contaminants vers les niveaux trophiques supérieurs.

\section{Remerciements}

Les auteurs remercient les rapporteurs pour leurs remarques constructives en vue de l'amélioration du manuscrit ainsi que les financeurs de la plateforme Recherche/Données/Informations du Plan Loire Grandeur Nature.

\section{RÉFÉRENCES}

Alomar H., Lemarchand C., Rosoux R., Vey D. \& Berny P. 2016. - Concentrations of organochlorine compounds (pesticides and $\mathrm{PCBs}$ ), trace elements ( $\mathrm{Pb}, \mathrm{Cd}, \mathrm{Cu}$, and $\mathrm{Hg}$ ), 134Cs, and 137Cs in the livers of the European otter (Lutra lutra), great cormorant (Phalacrocorax carbo), and European catfish (Silurus glanis), collected from the Loire River (France). European Journal of Wildlife Research 62 (6): 653-661. https://doi.org/10.1007/ s10344-016-1038-5

Berny P. J., Veniat A. \& MAZallon M. 2003. — Bioaccumulation of lead, cadmium, and lindane in zebra mussels (Dreissena polymorpha) and associated risk for bioconcentration in tufted duck (Aythia fuligula). Bulletin of Environmental Contamination and Toxicology 71: 90-97. https://doi.org/10.1007/s00128-003-0135-9

Binelli A., Galassi S. \& Provini A. 2001. — Factors affecting the use of Dreissena polymorpha as a bioindicator: The PCB pollution in lake Como (N. Italy). Water, Air, and Soil Pollution 125:
19-32. https://doi.org/10.1023/A:1005249822038

CAmusso M., BAlestrini R. \& Binelli A. 2001. — Use of zebra mussel (Dreissena polymorpha) to assess trace metal contamination in the largest Italian subalpine lakes. Chemosphere 44 (2): 263-270. https://doi.org/10.1016/S0045-6535(00)00169-7

Chevreuil M., Blanchard M., Teil M.-J., Carru A. M., TesTARD P. \& CHESTERIKOFF A. 1996. - Evaluation of the pollution by organochlorinated compounds (polychlorobiphenyls and pesticides) and metals $(\mathrm{Cd}, \mathrm{Cr}, \mathrm{Cu}$ and $\mathrm{Pb})$ in the water and in the zebra mussel (Dreissena polymorpha pallas) of the river Seine. Water, Air, and Soil Pollution 88: 371-381. https://doi. org/10.1007/BF00294112

Corvi C., Zimmerli P., Ortelli D., Khim-Heang S. \& Becker VAN SLOOTEN K. 2005. - Métaux et micropolluants organiques dans les eaux, les moules et les poissons du Léman. Rapport Commission internationale pour la Protection des Eaux du Léman contre la pollution, Campagne 2004, Duillier: 55-78.

Devin S., Bollache L., Nö̈l P.-Y. \& Beisel J.-N. 2005. — Patterns of biological invasions in French freshwater systems by non-indigenous macroinvertebrates. Hydrobiologia 551: 137-146. https://doi.org/10.1007/s10750-005-4456-z

Diaz-BARriga F., Borja-AbURTO V., WALISZEWKi S. \& YÁÑEZ L. 2003. - DDT in Mexico, in FiedLer H. (éd.), Persistent Organic Pollutants. The Handbook of Environmental Chemistry (Vol. 3 Series: Anthropogenic Compounds), vol 30. Springer, Berlin, Heidelberg: 371-388. https://doi.org/10.1007/10751132_14

Faria M., López M. A., Díez S. \& Barata C. 2010 . - Are native naiads more tolerant to pollution than exotic freshwater bivalve species? An hypothesis tested using physiological responses of three species transplanted to mercury contaminated sites in the Ebro River (NE, Spain). Chemosphere 81 (10): 1218-1226. https:// doi.org/10.1016/j.chemosphere.2010.09.037

GeNOVESI P. \& SHINE C. 2004. - European Strategy on Invasive Alien Species. Nature and Environment $n^{\circ} 137$. Council of Europe, Strasbourg, $67 \mathrm{p}$.

Gitahi S. M., Harper D. M., Muchiri S. M., Tole M. P. \& NG'ANG'A R. N. 2002. - Organochlorine and organophosphorus pesticide concentrations in water, sediment, and selected organisms in Lake Naivasha (Kenya). Hydrobiologia 488: 123-128. https://doi.org/10.1023/A:1023386732731

Jou L. J., Chen W. Y. \& LIAO C. M. 2009. - Online detection of waterborne bioavailable copper by valve daily rhythm in freshwater clam Corbicula fluminea. Environmental Monitoring and Assessment 155: 257-272. https://doi.org/10.1007/ s10661-008-0433-0

Kwan K. H. M., Chan H. M. \& Lafontaine Y. de 2003. - Metal contamination in zebra mussels (Dreissena polymorpha) along the St-Laurence River. Environmental Monitoring and Assessment 88: 193-219. https://doi.org/10.1023/A:1025517007605

LEMARChand C. 2007. - Etude de l'habitat de la Loutre d'Europe (Lutra lutra) en région Auvergne (France). Relations entre le régime alimentaire et la dynamique de composés essentiels et d'éléments toxiques. Thèse de Doctorat de l'Université Blaise-Pascal, ClermontFerrand, $225 \mathrm{p}$.

LEMARCHAND C., Rosoux R. \& Berny P. 2010. - Organochlorine pesticides, $\mathrm{PCB}$, heavy metals and anticoagulant rodenticides in tissues of Eurasian otters (Lutra lutra) from upper Loire River. Chemosphere 80 (10): 1120-1124. https://doi.org/10.1016/j. chemosphere.2010.06.026

Lemarchand C., Rosoux R. \& Berny P. 2011. - Semi aquatic top-predators as sentinels of diversity and dynamics of pesticides in aquatic food webs. The case of European otter (Lutra lutra) and Osprey (Pandion haliaetus) in Loire River catchment, France, in Stoytcheva M. (éd.), Pesticides in the Modern World: Risks and Benefits. InTech, Rijeka: 289-310.

LEMARCHAND C., Rosoux R. \& BERNy P. 2013a. - Étude écotoxicologique du bassin de la Loire à l'aide de bioindicateurs, dans le contexte des effets prévisibles des changements climatiques. Synthèse 
des résultats. Mémoire post-doctoral de VetAgro Sup, Plan Loire Grandeur Nature, Orléans, 98 p.

LeMARCHAND C., BeLlefroid M.-D.-N. DE \& Rosoux R. 2013b. Le Balbuzard pêcheur, histoire d'une sauvegarde. Catiche Productions, Nohanent, 32 p.

Lemarchand C., Rosoux R. \& Berny P. 2015. - Programme d'études écotoxicologiques concertées de bioindicateurs piscivores en Loire moyenne. Synthèse des résultats. VetAgro Sup, Catiche Productions, Plan Loire Grandeur Nature, Nohanent, 25 p.

LEMARCHAND C., AMBLARD C., SOUCHON Y. \& BERNY P. 2007. Organochlorine compounds (pesticides and pcbs) in scats of the European Otter (Lutra lutra) from an actual expanding population in central France. Water, Air and Soil Pollution 186: 55-62. https://doi.org/10.1007/s11270-007-9462-5

Lemarchand C., Rosoux R., Penide M.-E. \& Berny P. 2012. Tissue concentrations of pesticides, PCBs and metals among ospreys, (Pandion haliaetus), collected in France. Bulletin of Environmental Contamination and Toxicology 88: 89-93. https:// doi.org/10.1007/s00128-011-0453-2

Lemarchand C., Rosoux R., Talon C. \& Berny P. 2014. Flagship species conservation and introduced species invasion: toxic aspects along Loire River (France), in SOLONESKI S. (éd.) Pesticides - Toxic Aspects. InTech, Rijeka: 53-79. https://doi. org/10.5772/57176

Marie V., Baudrimont M. \& Boudou A. 2006. - Cadmium and zinc bioaccumultaion and metallothionein response in two freshwater bivalves (Corbicula fluminea and Dreissena polymorpha) transplanted along a polymetallic gradient. Chemosphere 65 (4): 609-617. https://doi.org/10.1016/j.chemosphere.2006.01.074

MaZeT A., KeCK G. \& BeRNY P. 2005. - Concentrations of PCBs, organochlorine pesticides and heavy metals (lead, cadmium and copper) in fish from the Drome river: Potential effects on otters (Lutra lutra). Chemosphere 61 (6): 810-816. https://doi. org/10.1016/j.chemosphere.2005.04.056

Pascal M., Lorvelec O. \& Vigne J.-D. 2006. - Invasions biologiques et extinctions: 11000 ans d'histoire des Vertébrés en France. Belin-Quæ, Paris, $350 \mathrm{p}$.

Prié V. 2017. - Nä̈ades et autres bivalves d'eau douce de France. Biotope, Mèze, 336 p.

Rosoux R. \& LEMARCHAND C. 2019. — La Loutre d'Europe. Biotope, Mèze, $352 \mathrm{p}$.

Schmitz D. C. \& Simberloff D. 1997. - Biological invasions: a growing threat. Issues in Science and Technology 13: 33-40.

SimON O. \& GARNIER-LAPLACE J. 2005. — Laboratory and field assessment of uranium trophic transfer efficiency in the crayfish Orconectes limosus fed the bivalve Corbicula fluminea. Aquatic Toxicology 74: 372-383. https://doi.org/10.1016/j.aquatox.2005.06.010

Simon O., Ribeyre F. \& Boudou A. 2000. - Comparative experimental study of cadmium and methylmercury trophic transfers between the Asiatic clam (Corbicula fluminea) and the crayfish (Astacus astacus). Archives of Environmental Contamination and Toxicology 38 (3): 317-326. https://doi.org/10.1007/ s002449910042

Sousa R., Antunes C. \& Guilhermo L. 2008. - Ecology of the invasive Asian clam Corbicula fluminea (Müller, 1774) in aquatic ecosystems: an overview. Annals of Limnology - International Journal ofLimnology 44 (2): 85-94. https://doi.org/10.1051/limn:2008017

Tablado Z., Tella J. L., Sánchez-Zapata J. A. \& Hiraldo F. 2010. - The paradox of the longterm positive effects of a North American Crayfish on an European community of predators. Conservation Biology 24 (5): 1230-1238. https://doi.org/10.1111/ j.1523-1739.2010.01483.x 